\title{
EULERIAN SERIES AS MODULAR FORMS REVISITED
}

\author{
ERIC T. MORTENSON
}

(Communicated by Kathrin Bringmann)

ABStract. Recently, Bringmann, Ono, and Rhoades employed harmonic weak Maass forms to prove results on Eulerian series as modular forms. By changing the setting to Appell-Lerch sums, we shorten the proof of one of their main theorems. In addition we discuss connections to recent work of Kang.

\section{Definitions And introduction}

Let $q$ be a complex number, $0<|q|<1$, and define $\mathbb{C}^{*}:=\mathbb{C}-\{0\}$. We recall

$$
\begin{aligned}
(x)_{n}=(x ; q)_{n} & :=\prod_{i=0}^{n-1}\left(1-q^{i} x\right), \quad(x)_{\infty}=(x ; q)_{\infty}:=\prod_{i \geq 0}\left(1-q^{i} x\right), \\
j(x ; q) & :=(x)_{\infty}(q / x)_{\infty}(q)_{\infty}=\sum_{n=-\infty}^{\infty}(-1)^{n} q^{\left(\begin{array}{c}
n \\
2
\end{array}\right)} x^{n},
\end{aligned}
$$

where in the last line the equivalence of product and sum follows from Jacobi's triple product identity. The following are special cases of the above definition. Let $a$ and $m$ be integers with $m$ positive. Define

$$
J_{a, m}:=j\left(q^{a} ; q^{m}\right), \quad \bar{J}_{a, m}:=j\left(-q^{a} ; q^{m}\right), \text { and } J_{m}:=J_{m, 3 m} .
$$

We will use the following definition of an Appell-Lerch sum. Using the notation of [10:

$$
m(x, q, z):=\frac{1}{j(z ; q)} \sum_{r=-\infty}^{\infty} \frac{(-1)^{r} q^{\left(\begin{array}{c}
r \\
2
\end{array}\right)} z^{r}}{1-q^{r-1} x z} .
$$

Appell-Lerch sums are useful in studying $q$-hypergeometric series [10, 15, 17. In original work of Lovejoy and Osburn [12,13, the results of Hickerson and the author on relating Hecke-type double sums to Appell-Lerch sums [10] were instrumental in determining mock theta behaviour of multisum $q$-hypergeometric series. One finds traces of Appell-Lerch sums throughout the Lost Notebook [16, where many identities express Eulerian series in terms of what are essentially $m(x, q, z)$ functions. For Ramanujan's sixth order mock theta functions $\phi(q)$ and $\sigma(q)$, one finds slightly rewritten [4, 16]:

$$
\phi(q):=\sum_{n=0}^{\infty} \frac{(-1)^{n} q^{n^{2}}\left(q ; q^{2}\right)_{n}}{(-q)_{2 n}}=2 m\left(q, q^{3},-1\right), \sigma(q):=\sum_{n=0}^{\infty} \frac{q^{\left(\begin{array}{c}
n+2 \\
2
\end{array}\right)(-q)_{n}}}{\left(q ; q^{2}\right)_{n+1}}=-m\left(q^{2}, q^{6}, q\right) .
$$

Received by the editors September 23, 2013 and, in revised form, January 21, 2014 and January 23,2014 .

2010 Mathematics Subject Classification. Primary 11B65, 11F11, 11F27.

Key words and phrases. Appell-Lerch sums, Eulerian forms, $q$-hypergeometric series. 
Appell-Lerch sums satisfy several well-known functional equations and identities, which we collect in the form of a proposition; see for example [10]. Here, the term generic means that the variables do not cause singularities in the Appell-Lerch sums or in the quotients of the theta functions.

Proposition 0.1. For generic $x, z, z_{0}, z_{1} \in \mathbb{C}^{*}$

$$
\begin{gathered}
m(x, q, z)=m(x, q, q z) \\
m(q x, q, z)=1-x m(x, q, z), \\
m\left(x, q, z_{1}\right)-m\left(x, q, z_{0}\right)=\frac{z_{0} J_{1}^{3} j\left(z_{1} / z_{0} ; q\right) j\left(x z_{0} z_{1} ; q\right)}{j\left(z_{0} ; q\right) j\left(z_{1} ; q\right) j\left(x z_{0} ; q\right) j\left(x z_{1} ; q\right)} .
\end{gathered}
$$

Although one does not find anything as explicit as (0.3a) - $0.3 \mathrm{c}$ in [16], one does find many specializations of the identities. For example, (0.3c) specializes to the following Lost Notebook relation for the above sixth orders [4, $(0.19)_{R}$ ]:

$$
\phi\left(q^{2}\right)+2 \sigma(q)=\prod_{n \geq 1}\left(1+q^{2 n-1}\right)^{2}\left(1-q^{6 n}\right)\left(1+q^{6 n-3}\right)^{2} .
$$

Another example is [2, Entry 12.4.1] which is a combination of ([10] 3.2b) and (0.3c). One can also view (0.4) as a linear combination of Eulerian series which essentially yields a weight $1 / 2$ weakly holomorphic modular form.

Ramanujan also expanded more involved Eulerian series in terms of AppellLerch-like sums. We recall [15, Proposition 2.6]:

$$
\begin{aligned}
\sum_{n=0}^{\infty} \frac{(-1)^{n} q^{n^{2}}\left(q ; q^{2}\right)_{n}}{\left(x ; q^{2}\right)_{n+1}\left(q^{2} / x ; q^{2}\right)_{n}} & =m(-x, q,-1)+\frac{J_{1,2}^{2}}{2 j(x ; q)}, \\
\left(1-\frac{1}{x}\right) \sum_{n=0}^{\infty} \frac{(-1)^{n}\left(q ; q^{2}\right)_{n} q^{(n+1)^{2}}}{\left(x q ; q^{2}\right)_{n+1}\left(q / x ; q^{2}\right)_{n+1}} & =m(-x, q,-1)-\frac{J_{1,2}^{2}}{2 j(x ; q)},
\end{aligned}
$$

where both are rewritten equations of [16] proved in Andrews [1. Identities such as (0.5)-(0.6) and the techniques of [10] are useful in finding additional $q$-hypergeometric and bilateral $q$-hypergeometric series with (mixed) mock modular behaviour [15].

In Ramanujan's last letter to Hardy, he included mock theta functions of orders three, five, and seven. The third orders could each be written as a special case of the generalised Lambert series $g(x, q)$. For example, take the third order $f(q)$ :

$$
f(q):=\sum_{n=0}^{\infty} \frac{q^{n^{2}}}{(-q)_{n}^{2}}=2-2 g(-1, q),
$$

where; see [10, Proposition 3.2]:

$$
\begin{aligned}
g(x, q): & =x^{-1}\left(-1+\sum_{n=0}^{\infty} \frac{q^{n^{2}}}{(x)_{n+1}(q / x)_{n}}\right)=\sum_{n=0}^{\infty} \frac{q^{n(n+1)}}{(x)_{n+1}(q / x)_{n+1}} \\
& =-x^{-1} m\left(q^{2} x^{-3}, q^{3}, x^{2}\right)-x^{-2} m\left(q x^{-3}, q^{3}, x^{2}\right) .
\end{aligned}
$$

Not until the discovery of the Lost Notebook and subsequent work of Andrews, Garvan, and Hickerson [3, 8, 9] was it realized that the fifth and seventh orders could each be expressed as the sum of a $g(x, q)$ and a single quotient of theta 
functions. These expressions for the fifth orders were the so-called mock theta conjectures. For the fifth order $f_{0}(q)$ :

$$
f_{0}(q):=\sum_{n=0}^{\infty} \frac{q^{n^{2}}}{(-q)_{n}}=-2 q^{2} g\left(q^{2}, q^{10}\right)+\frac{J_{5,10} J_{2,5}}{J_{1}} .
$$

In [10, Hickerson and the author developed and refined the notion of expanding Ramanujan's classical mock theta functions in terms of building blocks. We showed that if one allows repetition in $x$ in (0.2), one can always write these functions entirely in terms of $m(x, q, z)$ 's. If one does not allow for duplication in $x$, one can adjust the $z$ 's such that there is only a single quotient of theta functions. For $f_{0}(q)$ [10]:

$$
\begin{aligned}
f_{0}(q) & =m\left(q^{14}, q^{30}, q^{14}\right)+m\left(q^{14}, q^{30}, q^{29}\right)+q^{-2} m\left(q^{4}, q^{30}, q^{4}\right)+q^{-2} m\left(q^{4}, q^{30}, q^{19}\right) \\
& =2 m\left(q^{14}, q^{30}, q^{4}\right)+2 q^{-2} m\left(q^{4}, q^{30}, q^{4}\right)+\frac{J_{5,10} J_{2,5}}{J_{1}} .
\end{aligned}
$$

Such expansions are of interest when studying the partial theta function duals 15. To prove such expressions, we introduced the following Appell-Lerch sum identity.

Theorem $0.2([10])$. For generic $x, z, z^{\prime} \in \mathbb{C}^{*}$

$$
\begin{aligned}
& m(x, q, z)=\sum_{r=0}^{n-1} q^{-\left(\begin{array}{c}
r+1 \\
2
\end{array}\right)}(-x)^{r} m\left(-q^{\left(\begin{array}{c}
n \\
2
\end{array}\right)-n r}(-x)^{n}, q^{n^{2}}, z^{\prime}\right) \\
& +\frac{z^{\prime} J_{n}^{3}}{j(x z ; q) j\left(z^{\prime} ; q^{n^{2}}\right)} \sum_{r=0}^{n-1} \frac{q^{\left(\begin{array}{c}
r \\
2
\end{array}\right)}(-x z)^{r} j\left(-q^{\left(\begin{array}{c}
n \\
2
\end{array}\right)+r}(-x)^{n} z z^{\prime} ; q^{n}\right) j\left(q^{n r} z^{n} / z^{\prime} ; q^{n^{2}}\right)}{j\left(-q^{\left(\begin{array}{c}
n \\
2
\end{array}\right)}(-x)^{n} z^{\prime}, q^{r} z ; q^{n}\right)} .
\end{aligned}
$$

Independently, Gordon and McIntosh [7] expanded mock thetas in terms of multiple building blocks, but not in a comprehensive manner like (0.8). Also, no result like Theorem 0.2 was obtained. In fact, Theorem 0.2 and the $m(x, q, z)$ expansions in [10] enabled Lovejoy and Osburn [14] to give a short proof of conjectured identities for the tenth orders [7. They also note that since all classical mock theta functions can be written in terms of Appell-Lerch sums (see [10]), one can easily prove similar identities for second, third, sixth, and eighth orders; see [7, (5.2), (3.12), (5.10)] and the top of page 125 in [7].

\section{A FEW MORE TECHNiCAL DETAils}

We list a few more technical details [10. Some useful theta function identities are

$$
\bar{J}_{0,1}=2 \bar{J}_{1,4}=\frac{2 J_{2}^{2}}{J_{1}}, \bar{J}_{1,2}=\frac{J_{2}^{5}}{J_{1}^{2} J_{4}^{2}}, J_{1,2}=\frac{J_{1}^{2}}{J_{2}}, \bar{J}_{1,3}=\frac{J_{2} J_{3}^{2}}{J_{1} J_{6}}, J_{1,4}=\frac{J_{1} J_{4}}{J_{2}} .
$$

We state additional general identities for the theta function:

$$
\begin{gathered}
j\left(q^{n} x ; q\right)=(-1)^{n} q^{-\left(\begin{array}{c}
n \\
2
\end{array}\right)} x^{-n} j(x ; q), \quad n \in \mathbb{Z}, \\
j(x ; q)=j(q / x ; q)=-x j\left(x^{-1} ; q\right), \\
j(x ; q)=J_{1} j\left(x, q x ; q^{2}\right) / J_{2}^{2},
\end{gathered}
$$




$$
\begin{gathered}
j(z ; q)=j\left(-q z^{2} ; q^{4}\right)-z j\left(-q^{3} z^{2} ; q^{4}\right), \\
j\left(x^{2} ; q^{2}\right)=j(x ; q) j(-x ; q) / J_{1,2} .
\end{gathered}
$$

$$
\text { rocal of Jacobi's theta product: }
$$

$$
\sum_{n=-\infty}^{\infty} \frac{(-1)^{n} q^{\left(\begin{array}{c}
n+1 \\
2
\end{array}\right)}}{1-q^{n} z}=\frac{J_{1}^{3}}{j(z ; q)}
$$

Finally we note for generic $x, y, z \in \mathbb{C}^{*}$ :

$$
j(x ; q) j(y ; q)=j\left(-x y ; q^{2}\right) j\left(-q x^{-1} y ; q^{2}\right)-x j\left(-q x y ; q^{2}\right) j\left(-x^{-1} y ; q^{2}\right) .
$$

\section{The TheOrem And THE ALternate Proof}

Motivated by Dyson's rank differences, Bringmann, Ono, and Rhoades [5] used the theory of harmonic weak Maass forms in order to identify linear combinations of Eulerian series which are weakly holomorphic modular forms. by

We recall the relevant notation from [5]. Define $K^{\prime}(w ; z), K^{\prime \prime}(w ; z), H^{\prime}(a, c, w ; z)$,

$$
\begin{aligned}
K^{\prime}(\omega ; z) & :=\sum_{n=0}^{\infty} \frac{(-1)^{n} q^{n^{2}}\left(q ; q^{2}\right)_{n}}{\left(\omega q^{2} ; q^{2}\right)_{n}\left(\omega^{-1} q^{2} ; q^{2}\right)_{n}}, \\
K^{\prime \prime}(\omega ; z) & :=\sum_{n=1}^{\infty} \frac{(-1)^{n} q^{n^{2}}\left(q ; q^{2}\right)_{n-1}}{\left(\omega q ; q^{2}\right)_{n}\left(\omega^{-1} q ; q^{2}\right)_{n}}, \\
H^{\prime}(a, c, w ; z) & :=\sum_{n=0}^{\infty} \frac{q^{\frac{1}{2} n(n+1)}(-q)_{n}}{\left(\omega q^{\frac{a}{c}}\right)_{n+1}\left(\omega q^{1-\frac{a}{c}}\right)_{n+1}},
\end{aligned}
$$

where $q:=e^{2 \pi i z}$ and $0<a<c$. Further, let $\zeta_{c}:=e^{2 \pi i / c}$ and $f_{c}:=2 c / \operatorname{gcd}(c, 4)$. Let

$$
\begin{array}{r}
\tilde{K}(a, c ; z):=\frac{1}{4} \csc \left(\pi \frac{a}{c}\right) q^{-\frac{1}{8}} K^{\prime}\left(\zeta_{c}^{a} ; z\right)+\sin \left(\pi \frac{a}{c}\right) q^{-\frac{1}{8}} K^{\prime \prime}\left(\zeta_{c}^{a} ; z\right), \\
\tilde{H}(a, c ; z):=q^{\frac{a}{c}\left(1-\frac{a}{c}\right)}\left(H^{\prime}(a, c, 1 ; z)-H^{\prime}(a, c,-1 ; z)\right),
\end{array}
$$

where a sign error has been corrected in (2.5). One of the main results of [5] reads

Theorem 2.1 ([5, Theorem 1.3]). Let $0<a<c$. In the notation above, $\tilde{H}\left(a, c ; 4 f_{c}^{2} z\right)$ is a weight $1 / 2$ weakly holomorphic modular form on $\Gamma_{1}\left(64 f_{c}^{4}\right)$ and $\tilde{K}\left(a, c ; 2 f_{c}^{2} z\right)$ is a weight $1 / 2$ weakly holomorphic modular form on $\Gamma_{1}\left(64 f_{c}^{4}\right)$.

The proof of Theorem 2.1] is lengthy and detailed and yields no explicit formulas for $\tilde{H}$ and $\tilde{K}$. Here, we change the context to Appell-Lerch sums and employ the techniques of [10] to shorten the proof of Theorem 2.1 and discuss connections to recent work of Kang [11].

Theorem 2.2. Let $0<a<c$. In the notation above, we have

$$
\tilde{H}(a, c ; z)=2 q^{\frac{a}{c}\left(1-\frac{a}{c}\right)} \frac{J_{2}^{3}}{J_{1,2} j\left(q^{\frac{2 a}{c}} ; q^{2}\right)}, \quad \tilde{K}(a, c ; z)=-\frac{i \zeta_{c}^{a / 2} q^{-\frac{1}{8}}}{2} \frac{J_{1,2}^{2}}{j\left(\zeta_{c}^{a} ; q\right)} .
$$

For Theorem 2.2 we give two proofs of the explicit expressions for $\tilde{K}$. The first uses (0.5) and (0.6) while the second demonstrates how to use new Appell-Lerch sum properties to go from the Watson-Whipple results of Kang [1] to identities (0.5) and (0.6). 
Proof of Theorem 2.2. Using (0.5) and (0.6), we see

$$
\begin{aligned}
K^{\prime}(\omega ; z) & =(1-\omega)\left(m(-\omega, q,-1)+\frac{J_{1,2}^{2}}{2 j(\omega ; q)}\right), \\
K^{\prime \prime}(\omega ; z) & =\frac{\omega}{1-\omega}\left(m(-\omega, q,-1)-\frac{J_{1,2}^{2}}{2 j(\omega ; q)}\right) .
\end{aligned}
$$

The explicit form for $\tilde{K}$ is then immediate upon writing sine and cosecant to exponential form. This completes the first proof.

For the second proof, we first note that Kang [11 showed via Watson-Whipple [6. eq. (2.5.1), p. 43]:

$$
\begin{gathered}
\frac{1}{1-\omega} K^{\prime}(\omega ; \tau)=\frac{1}{\bar{J}_{1,4}} \sum_{n=-\infty}^{\infty} \frac{q^{2 n^{2}+n}}{1-\omega q^{2 n}}, \\
\left(1-\frac{1}{\omega}\right) K^{\prime \prime}(\omega ; \tau)=-\frac{1}{\bar{J}_{1,4}} \sum_{n=-\infty}^{\infty} \frac{q^{2 n^{2}+3 n+1}}{1-\omega q^{2 n+1}} .
\end{gathered}
$$

In 11, one adds (2.6) and (2.7) and uses (1.3). Identity (2.6) is also in 1, 16. We show how to use Theorem 0.2 and elementary theta function properties to obtain (0.5) from (2.6). The proof for (0.6) is similar. We begin with

$$
\begin{aligned}
\frac{1}{\bar{J}_{1,4}} \sum_{n=-\infty}^{\infty} \frac{q^{2 n^{2}+n}}{1-\omega q^{2 n}} & =\frac{1}{\bar{J}_{1,4}} \sum_{n=-\infty}^{\infty} \frac{q^{2 n^{2}+n}}{1-\omega^{2} q^{4 n}}+\frac{\omega}{\bar{J}_{1,4}} \sum_{n=-\infty}^{\infty} \frac{q^{2 n^{2}+3 n}}{1-\omega^{2} q^{4 n}} \\
& =m\left(-\omega^{2} q, q^{4},-q^{3}\right)+\omega q^{-1} m\left(-\omega^{2} q^{-1}, q^{4},-q\right) \\
& =m\left(-\omega^{2} q, q^{4},-q\right)+\omega q^{-1} m\left(-\omega^{2} q^{-1}, q^{4},-q\right)+\frac{J_{1,2}^{2} j\left(-q \omega^{2} ; q^{4}\right)}{j(\omega ; q) j(-\omega ; q)},
\end{aligned}
$$

where we have used (1.2a), (1.2b), (1.2c), (1.2e), (0.3a), (10.3c) and simplified. Using Theorem 0.2 with $n=2, x=-\omega, z=-1$, and $z^{\prime}=-q$ and more simplifying we obtain

$$
\begin{aligned}
\frac{1}{\bar{J}_{1,4}} \sum_{n=-\infty}^{\infty} \frac{q^{2 n^{2}+n}}{1-\omega q^{2 n}}= & m(-\omega, q,-1)+\frac{J_{1,2}^{2} j\left(-q \omega^{2} ; q^{4}\right)}{j(\omega ; q) j(-\omega ; q)} \\
& -\frac{J_{1,2}^{2}}{2 j(\omega ; q)^{2} j(-\omega ; q)}\left[j\left(-\omega^{2} ; q^{2}\right) \bar{J}_{1,2}-\omega j\left(-q \omega^{2} ; q^{2}\right) \bar{J}_{0,2}\right] \\
= & m(-\omega, q,-1)+\frac{J_{1,2}^{2} j\left(-q \omega^{2} ; q^{4}\right)}{j(\omega ; q) j(-\omega ; q)}-\frac{J_{1,2}^{2}}{2 j(-\omega ; q)} \\
= & m(-\omega, q,-1)+\frac{J_{1,2}^{2}}{2 j(-\omega ; q) j(\omega ; q)}\left[2 j\left(-q \omega^{2} ; q^{4}\right)-j(\omega ; q)\right] \\
= & m(-\omega, q,-1)+\frac{J_{1,2}^{2}}{2 j(-\omega ; q) j(\omega ; q)}\left[j\left(-q \omega^{2} ; q^{4}\right)+\omega j\left(-q^{3} \omega^{2} ; q^{4}\right)\right] \\
= & m(-\omega, q,-1)+\frac{J_{1,2}^{2}}{2 j(\omega ; q)},
\end{aligned}
$$

where the second equality follows from (1.4) and the last two from (1.2d). 
For $\tilde{H}$, we begin with [5, (4.1)]:

$$
\begin{aligned}
H(a, b, c ; z): & =\frac{1}{J_{1,2}} \sum_{n=-\infty}^{\infty} \frac{(-1)^{n} q^{n+\frac{a}{c}} q^{n(n+1)}}{1-\zeta_{c}^{b} q^{n+\frac{a}{c}}} \\
& =\frac{q^{\frac{a}{c}}}{J_{1,2}} \sum_{n=-\infty}^{\infty} \frac{(-1)^{n} q^{n^{2}+2 n}}{1-\zeta_{c}^{2 b} q^{\frac{2 a}{c}} q^{2 n}}+\frac{\zeta_{c}^{b} q^{\frac{2 a}{c}}}{J_{1,2}} \sum_{n=-\infty}^{\infty} \frac{(-1)^{n} q^{n^{2}+3 n}}{1-\zeta_{c}^{2 b} q^{\frac{2 a}{c}} q^{2 n}} \\
& =-q^{\frac{a}{c}-1} m\left(\zeta_{c}^{2 b} q^{\frac{2 a}{c}-1}, q^{2}, q\right)+\frac{\zeta_{c}^{-b} J_{2}^{3}}{J_{1,2} j\left(\zeta_{c}^{2 b} q^{\frac{2 a}{c}} ; q^{2}\right)},
\end{aligned}
$$

where in the last line we used (0.2) and (1.3). The result follows upon recalling [5. (4.5)]

$$
\tilde{H}(a, c ; z)=q^{\frac{a}{c}\left(1-\frac{a}{c}\right)}(H(a, 0, c ; z)-H(a, c / 2, c ; z)) .
$$

\section{ACKNOWLEDGEMENTS}

We would like to thank Wadim Zudilin and the referee for helpful comments and suggestions.

\section{REFERENCES}

[1] George E. Andrews, Mordell integrals and Ramanujan's "lost" notebook, Analytic number theory (Philadelphia, Pa., 1980), Lecture Notes in Math., vol. 899, Springer, Berlin, 1981, pp. 10-18. MR654518 (83m:33004)

[2] George E. Andrews and Bruce C. Berndt, Ramanujan's lost notebook. Part I, Springer, New York, 2005. MR2135178(2005m:11001)

[3] George E. Andrews and F. G. Garvan, Ramanujan's "lost" notebook. VI. The mock theta conjectures, Adv. in Math. 73 (1989), no. 2, 242-255, DOI 10.1016/0001-8708(89)90070-4. MR 987276 (90d:11115)

[4] George E. Andrews and Dean Hickerson, Ramanujan's "lost" notebook. VII. The sixth order mock theta functions, Adv. Math. 89 (1991), no. 1, 60-105, DOI 10.1016/0001-8708(91)90083J. MR.1123099(92i:11027)

[5] Kathrin Bringmann, Ken Ono, and Robert C. Rhoades, Eulerian series as modular forms, J. Amer. Math. Soc. 21 (2008), no. 4, 1085-1104, DOI 10.1090/S0894-0347-07-00587-5. MR2425181(2010a:11078)

[6] George Gasper and Mizan Rahman, Basic hypergeometric series, 2nd ed., Encyclopedia of Mathematics and its Applications, vol. 96, Cambridge University Press, Cambridge, 2004. With a foreword by Richard Askey. MR2128719 (2006d:33028)

[7] Basil Gordon and Richard J. McIntosh, A survey of classical mock theta functions, Partitions, $q$-series, and modular forms, Dev. Math., vol. 23, Springer, New York, 2012, pp. 95-144, DOI 10.1007/978-1-4614-0028-8_9. MR3051186

[8] Dean Hickerson, A proof of the mock theta conjectures, Invent. Math. 94 (1988), no. 3, 639660, DOI 10.1007/BF01394279. MR969247(90f:11028a)

[9] Dean Hickerson, On the seventh order mock theta functions, Invent. Math. 94 (1988), no. 3, 661-677, DOI 10.1007/BF01394280. MR969248(90f:11028b)

[10] Dean R. Hickerson and Eric T. Mortenson, Hecke-type double sums, Appell-Lerch sums, and mock theta functions, I, Proc. Lond. Math. Soc. (3) 109 (2014), no. 2, 382-422, DOI 10.1112/plms/pdu007. MR 3254929

[11] Soon-Yi Kang, Mock Jacobi forms in basic hypergeometric series, Compos. Math. 145 (2009), no. 3, 553-565, DOI 10.1112/S0010437X09004060. MR2507741 (2010f:33022)

[12] Jeremy Lovejoy and Robert Osburn, The Bailey chain and mock theta functions, Adv. Math. 238 (2013), 442-458, DOI 10.1016/j.aim.2013.02.005. MR 3033639

[13] Jeremy Lovejoy and Robert Osburn, q-hypergeometric double sums as mock theta functions, Pacific J. Math. 264 (2013), no. 1, 151-162, DOI 10.2140/pjm.2013.264.151. MR3079764

[14] J. Lovejoy, R. Osburn, On two 10th order mock theta identities, Ramanujan Journal, to appear, arxiv:1209.2315. 
[15] Eric T. Mortenson, On the dual nature of partial theta functions and Appell-Lerch sums, Adv. Math. 264 (2014), 236-260, DOI 10.1016/j.aim.2014.07.018. MR3250284

[16] Srinivasa Ramanujan, The lost notebook and other unpublished papers, Springer-Verlag, Berlin, 1988. With an introduction by George E. Andrews. MR.947735 (89j:01078)

[17] S. P. Zwegers, Mock theta functions, Ph.D. Thesis, Universiteit Utrecht, 2002.

Max-Planck-Institut für Mathematik, Vitvatsgasse 7, 53111 Bonn, Germany

E-mail address: etmortenson@gmail.com 